\title{
Day Times Millimole per Liter per Kilogram per Meter Squared
}

National Cancer Institute

\section{Source}

National Cancer Institute. Day Times Millimole per Liter per Kilogram per Meter Squared. NCI Thesaurus. Code C111177.

Days times millimoles per liter, divided by kilograms per meter squared. 